\title{
Resolution Enhancement by Incorporating Segmentation-based Optical Flow Estimation
}

\author{
Osama A. Omer \\ Department of Electrical Engineering \\ South Valley University \\ Aswan, Egypt
}

\begin{abstract}
In this paper, the problem of recovering a highresolution frame from a sequence of low-resolution frames is considered. High-resolution reconstruction process highly depends on image registration step. Typical resolution enhancement techniques use global motion estimation technique. However, in general, video frames cannot be related through global motion due to the arbitrary individual pixel movement between frame pairs. To overcome this problem, we propose to employ segmentation-based optical flow estimation technique for motion estimation with a modified model for frame alignment. To do that, we incorporate the segmentation with the optical flow estimation in two-stage optical flow estimation. In the first stage, a reference image is segmented into homogeneous regions. In the second stage, the optical flow is estimated for each region rather than pixels or blocks. Then, the frame alignment is accomplished by optimizing the cost function that consists of $L_{1}$-norm of the difference between the interpolated low-resolution (LR) frames and the simulated LR frames. The experimental results demonstrate that using segmentation-based optical flow estimation in motion estimation step with the modified alignment model works better than other motion models such as affine, and conventional optical flow motion models.
\end{abstract}

Keywords- Optical flow; image segmentation; Horn-Schunck; super resolution; resolution enhancement.

\section{INTRODUCTION}

Multi-frame super resolution (SR) is the process of producing a resolution-enhanced frame from multiple lowresolution (LR) frames with sub-pixel shift. SR received much attention in computer vision and image processing communities over the past three decades [1-21] (for review see [6]). SR process typically includes three steps: (i) image registration (motion estimation), (ii) the alignment of LR frames on the high-resolution (HR) grid, and (iii) image restoration.

SR methods can be categorized, based on the domain in which the process is done, into time domain [1-5, 7-8, 10-21] and frequency domain [9]. In another way, they can be categorized, based on the incorporated motion model, into global motion model (including translation [3] and affine [2] models) and local motion model (including optical flow [7, 12] and block matching [13]). Furthermore, they can be categorized, based on the alignment process, into non-uniform interpolation [8], deterministic and stochastic regularization [10], and projection onto convex sets (POCS) [11].
Most of the existing super-resolution algorithms [2-3, 911] cannot cope with local motion, because they assume that motion model can be globally parameterized. To overcome the problems of registration error in locally moving parts, three techniques appeared in the literature. The first is to use different global (or local) weights for different registration error levels $[14,16]$. The second is to use local motion (or multi-motion) estimation to improve the accuracy of registration in the locally moving parts [7, 12, 15, 19]. The third is a combination of the previous two techniques $[17,18]$. Among these techniques, the first technique is widely used in SR for its simplicity. The idea of using different weights for different registration is based on rejecting pixels or even whole frames that have high registration error. On the other hand, the main idea behind using local motion estimation techniques is to incorporate information from different frames as much as possible $[12,15,19]$. The main problem of multimotion estimation [19] is the complexity, since it requires estimation of motion for each moving object in all frames, which is complex and not always accurate because of the fact that the motion of different object affects the motion of other objects. Also, using conventional optical flow estimation for motion estimation [7, 12] is sensitive to noise. In addition, using block-matching results in blocking artefacts due to dividing frames into blocks and incorrectly assuming that all pixels within each block have the same motion vector. Moreover, using combination of local motion estimation and weighting technique adds more complexity to the algorithm as proposed in $[17,18]$. In addition, algorithm proposed in [15] requires high computational time, since it perform region matching using full search.

On the other hand, region segmentation has been employed for image SR in [14, 15, 20, 21]. In [14], segmentation is employed in a region rejection based on the registration inaccuracies. While in [15], region is employed in a region matching in the registration step. In [20], a region-based super-resolution algorithm is proposed in which different filters are used according to the type of region. In this method the segmentation information is not fully used where it is used only to classify regions into homogeneous and inhomogeneous regions. In [21], the image is segmented into background and different objects and each of these are super-resolved separately using a traditional technique and then the superresolved regions are merged to construct the HR image. This algorithm is very complex since it requires segmentation of moving objects and registration of each object separately. 
In order to overcome these difficulties, we propose a modified gradient-based optical flow estimation algorithm based on the Horn-Schunck algorithm, so that tries to overcome the problems of conventional optical flow estimation by the use of image segmentation.

Based on the assumption that pixels in a homogeneous arbitrary shaped region have the same motion then motion discontinuities go along with discontinuities in the intensity image. In addition, using segmentation reduces the susceptibility to noise. Furthermore, we propose to incorporate the modified gradient-based optical flow estimation algorithm for estimating motion of each region in image registration.

Our claim in this work is that the enhancement of the motion estimation results leads to enhancement in the SR results. Therefore, we establish a framework of multi-frame SR method that utilizes segmentation-based optical flow estimation.

The method in the proposed framework is summarized as follows. First, the frames are interpolated to make optical flow estimation in sub-pixel accuracy, and then the interpolated reference frame is segmented into arbitrary shaped regions using watershed transform [22].

The locally moving segments are then motion compensated using search. The next step is image alignment, where we fuse the motion compensated frames to produce one blurred HR frame by using the $\mathrm{L}_{1}$-norm in the frame alignment step. The so generated HR frame is de-blurred by using a regularization-based restoration method.

\section{PROBLEM DESCRIBTION}

The problem of multi-frame SR is to estimate a highresolution frame out of observed successive LR frames. Assume that N LR frames of the same scene denoted by $\mathrm{Y}^{\mathrm{k}}(1$ $\leq \mathrm{k} \leq \mathrm{N}$ ), each containing $\mathrm{M}^{2}$ pixels, are observed, and they are generated from the HR frame denoted by $\mathrm{X}$, containing $\mathrm{L}^{2}$ pixels, where $\mathrm{L} \geq \mathrm{M}$. The observation of $\mathrm{N}$ LR frames are modelled as follows:

$$
\mathrm{Y}^{\mathrm{k}}=\mathbf{D H} \mathbf{F}^{\mathrm{k}} \mathrm{X}+\mathrm{V}^{\mathrm{k}}
$$

where $\mathbf{F}^{\mathrm{k}}, \mathbf{H}$ and $\mathbf{D}$ are the motion, blurring, and downsampling operators, respectively. The size of $\mathbf{F}^{\mathrm{k}}, \mathbf{H}$ and $\mathbf{D}$ are $\mathrm{L}^{2} \times \mathrm{L}^{2}, \mathrm{~L}^{2} \times \mathrm{L}^{2}$ and $\mathrm{M}^{2} \times \mathrm{L}^{2}$, respectively. $\mathrm{X}$ is the unknown $\mathrm{HR}$ frame, $\mathrm{Y}^{\mathrm{k}}$ is the $\mathrm{k}$-th observed LR frame, and $\mathrm{V}^{\mathrm{k}}$ is an additive random noise for the $\mathrm{k}$-th frame with the same size as $\mathrm{Y}^{\mathrm{k}}$.

Throughout the paper, we assume that $\mathbf{D}$ and $\mathbf{H}$ are known and the additive noise is Gaussian. The problem here in this paper is to estimate the motion for each frame, $\mathbf{F}^{\mathrm{k}}$, and to find the original image, $\mathrm{X}$.

The assumption of known and constant down-sampling operator is a practical assumption, since in resolution enhancement applications, the enhancement factor is determined by the user and then the down-sampling operator is known. Also, even if in practical applications, the blurring operator is not known, it can be estimated by any of the blur estimation algorithms for example see [27].

\section{MODIFIED HORN-SCHUNCK OPTICAL FLOW ESTIMATION}

In the conventional Horn-Schunck optical flow estimation algorithm, an equation that relates the changes in image brightness at a point to the motion of the brightness pattern has been derived. Assuming that the image brightness at the point $(\mathrm{x}, \mathrm{y})$ in the image plane at time $\mathrm{t}$ be denoted by $\mathrm{E}(\mathrm{x}, \mathrm{y}, \mathrm{t})$. The brightness of a particular point in the pattern is constant, so that

$$
\frac{\mathrm{dE}}{\mathrm{dt}}=0
$$

Then, by using chain rule for differentiation, it becomes

$$
\mathrm{E}_{\mathrm{x}} \mathrm{u}+\mathrm{E}_{\mathrm{y}} \mathrm{v}+\mathrm{E}_{\mathrm{t}}=0
$$

Where $\mathrm{u}=\frac{\partial \mathrm{x}}{\partial \mathrm{t}}, \mathrm{v}=\frac{\partial \mathrm{y}}{\partial \mathrm{t}}, \mathrm{E}_{\mathrm{x}}=\frac{\partial \mathrm{E}}{\partial \mathrm{x}}, \mathrm{y}=\frac{\partial \mathrm{E}}{\partial \mathrm{y}}, \mathrm{E}_{\mathrm{t}}=\frac{\partial \mathrm{E}}{\partial \mathrm{t}}$. To allow smoothness of the optical flow, the smoothness measure term is added in the minimization process. The Laplacian of the $\mathrm{x}$ and y-components of the flow are used as the smoothness measure function. So that the optical flow estimation problem is described as the minimization of

$$
\varepsilon^{2}=\varepsilon_{\mathrm{b}}^{2}+\alpha^{2} \varepsilon_{\mathrm{c}}^{2},
$$

Where

$$
\begin{gathered}
\varepsilon_{b}=E_{x} u+E_{y} v+E_{t} \\
\varepsilon_{c}^{2}=\left(\frac{\partial u}{\partial x}\right)^{2}+\left(\frac{\partial u}{\partial y}\right)^{2}+\left(\frac{\partial v}{\partial x}\right)^{2}+\left(\frac{\partial v}{\partial y}\right)^{2}
\end{gathered}
$$

$\alpha$ is the weighting parameter that weights the importance between $\varepsilon_{\mathrm{b}}$ and $\varepsilon_{\mathrm{c}}$. This problem is solved iteratively as follow

$$
\begin{gathered}
u^{(n+1)}=u^{(n)}-E_{x}\left[E_{x} u\left({ }^{(n)}+E_{y} v^{(n)}+E_{t}\right] /\left[\alpha^{2}+E_{x}^{2}\right.\right. \\
\left.+E_{y}^{2}\right]
\end{gathered}
$$

And

$$
\begin{gathered}
v^{(n+1)}=v^{(n)}-E_{y}\left[E_{x} u^{(n)}+E_{y} v^{(n)}+E_{t}\right] /\left[\alpha^{2}+E_{x}^{2}\right. \\
\left.+E_{y}^{2}\right]
\end{gathered}
$$

Where $\mathrm{n}$ is the iteration number.

The main modification of the Horn-Schunck algorithm is represented by the incorporation of the segmentation information in the optical flow estimation, which done in two stages. The two stages are described as follows.

\section{A. Image Segmentation}

Without loss of generality, we used the watershed transform for image segmentation in this work. The watershed transform is a region segmentation approach, in which the image is supposed to take high gradient values in the neighbourhood of edges and low gradient values for interior pixels. The segmentation can be obtained by removing some 
of weakest edges, which will create a number of lakes by grouping all the pixels that lie below a certain threshold. This can reduce the influence of noise and reduce the oversegmentation problem. It is then determined for each pixel in which direction the rain would flow if it would fall on the topographic activity surface. The segmentation process is done for the reference frame only to reduce the computational complexity.

The main steps of the watershed segmentation algorithm are summarized as follows (see [22] for details):

1) Define the floating point activity as $|\nabla f|=\sqrt{f_{x}^{2}+f_{y}^{2}}$, where $\left(f_{x} ; f_{y}\right)$ is image gradient,

2) Assign a label for each pixel position,

3) For each pixel position find the weak edges, in which the floating point activity value is less than certain threshold $(\mu)$ (the thresholding is done for the floating point activity in the positions ep; swp; sp and sep as defined in Fig. 1),

4) Remove these weak edges by merging the corresponding labels,

5) For further merge regions and remove the effect of noise do; for each pixel position find the direction of rainfalling among the eight directions (direction of rainfalling is defined as the direction which has the smallest difference between the floating point activity of the central position (cp) and the other position (nwp; np; nep;wp; ep; swp; sp or sep),

6) Finally, the pixels that have the same label belong to the same region.

An example for segmented image is shown in Fig. 2. This figure shows the suitability of the watershed segmentation algorithm.

\begin{tabular}{|l|l|l|}
\hline nwp & np & nep \\
\hline wp & cp & ep \\
\hline swp & sp & sep \\
\hline
\end{tabular}

Figure 1: A diagram showing central pixel and neighboring pixels.

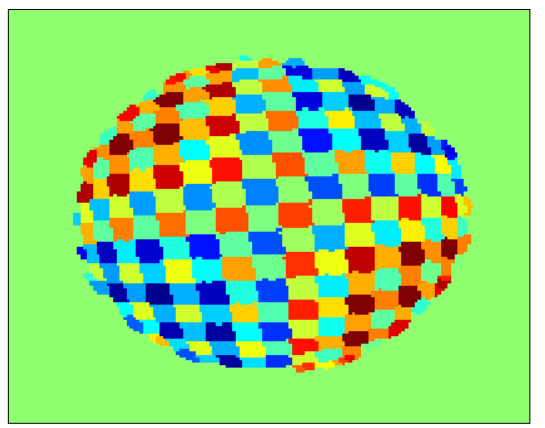

Figure 2: An example for segmented image form rotation sequence.

\section{B. Segmentation-based Horn-Schunck Algorithm}

As Fig. 2 shows an example for the segmented image where each colour represents a homogeneous region, our assumption is that regions' discontinuity is the motion discontinuity. So that, rather than estimating optical flow for each pixel as in $[23,24,25]$ or estimation a single optical flow for each block by assuming that all pixels in a certain block [26] have the same motion, we assume that motion of all pixels in each homogeneous region is the same. Therefore, we suggest modifying Eqs. (3) and (4) to be

$$
\begin{array}{rlrl}
u_{\mathfrak{R}}^{(n+1)}=u_{\mathfrak{R}}^{(n)}- & \operatorname{median}_{x, y}\left\{E _ { x } \left[E_{x} u^{(n)}\right.\right. & \\
& \left.+E_{y} v^{(n)}+E_{t}\right] /\left[\alpha^{2}+E_{x}^{2}\right. & & \forall x, y \\
& \left.\left.+E_{y}^{2}\right]\right\} & & \in \mathfrak{R}
\end{array}
$$

And

$$
\begin{aligned}
& v_{\Re}^{(n+1)}=v_{\Re}^{(n)}-\text { median }_{x, y}\left\{E _ { y } \left[E_{x} u^{(n)}\right.\right. \\
& \begin{array}{ll}
\left.+E_{y} v^{(n)}+E_{t}\right] /\left[\alpha^{2}+E_{x}^{2}\right. & \forall x, y \\
& \in \mathfrak{R}
\end{array} \\
& \left.\left.+E_{y}^{2}\right]\right\}
\end{aligned}
$$

Where $\left(u_{\Re}^{(n+1)}, v_{\Re}^{(n+1)}\right)$ is the motion vector for all pixels in the region $\Re, \operatorname{median}_{\mathrm{x}, \mathrm{y}}\{$.$\} is the median value evaluation$ function. The median value is estimated done for all values of $\mathrm{x}$, $\mathrm{y}$ in region $\mathfrak{R}$. Here we suggested using median function for its robustness against noise.

\section{RESOLUTION ENHANCEMENT BASED ON MODIFIED OPTICAL FLOW ESTIMATION}

In this section, we illustrate the method for optimizing a cost function that consists of the error between the simulated LR frames and the interpolated observed LR frames.

The proposed cost function incorporates the effect of local motion.

\section{A. Cost Function}

We start with the resolution enhancement problem which can be described as an optimization problem as follows:

$$
\mathrm{J}_{1}[\mathrm{X}]=\sum_{\mathrm{k}=1}^{\mathrm{N}}\left\|\mathbf{H F}^{\mathrm{k}} \mathrm{X}-\widetilde{\mathrm{Y}}^{\mathrm{k}}\right\|_{1}
$$

where $\|.\|_{1}$ is the $\mathrm{L}_{1}$-norm which describes the cost function measuring error and $\widetilde{\mathrm{Y}}^{\mathrm{k}}$ is defined as the upsampled and interpolated frame from the observed LR frame $\mathrm{Y}^{\mathrm{k}}$ :

$$
\widetilde{\mathrm{Y}}^{\mathrm{k}}=\mathcal{J}\left(\mathbf{D}^{\mathrm{T}} \mathrm{Y}^{\mathrm{k}}\right)
$$

where $\mathcal{J}($.$) is the interpolation operator, which is defined$ on the missed pixels positions only.

On the other hand, the general traditional cost function directly comes from $(8)$ is $[2,4]$

$$
\mathrm{J}_{2}[\mathrm{X}]=\sum_{\mathrm{k}=1}^{\mathrm{N}}\left\|\mathbf{D H} \mathbf{F}^{\mathrm{k}} \mathrm{X}-\mathrm{Y}^{\mathrm{k}}\right\|_{1}
$$

This cost function is so ill-posed that we need to add a regularization term in practice. Indeed, $\mathrm{J}_{1}[\mathrm{X}]$ and $\mathrm{J}_{2}[\mathrm{X}]$ are related and $\mathrm{J}_{1}[\mathrm{X}]$ includes "less" ill-posedness than $\mathrm{J}_{2}[\mathrm{X}]$ does as proved in [15]. The cost function $J_{1}[X]$ is modified as follows

$$
\mathrm{J}_{3}[\mathrm{X}]=\sum_{\mathrm{k}=1}^{\mathrm{N}}\left\|\mathbf{H F}^{\mathrm{k}} \mathrm{X}-\widetilde{\mathrm{Y}}^{\mathrm{k}}\right\|_{1}+\lambda\|\mathbf{C X}\|_{1}
$$

Where $\mathbf{C}$ is a matrix that represents a Laplacian high pass operator and $\lambda$ is the regularization parameter. The regularization term in (10) is added to solve the ill-posedness 
of the inverse problem. The regularization term incorporates the smoothness property of the HR frame.

\section{B. Two Steps-Based Minimization of $J_{3}[X]$}

The reconstruction of the $\mathrm{HR}$ image $(\mathrm{X})$ can be divided into two independent steps, namely, the fusion and restoration steps. The fusion problem is described as

$$
\hat{Z}=\arg \min _{Z} \sum_{k=1}^{N}\left\|F_{k} Z-\tilde{Y}_{k}\right\|_{1}
$$

This can be obtained by median filtering as:

$$
\widehat{Z}(i, j)=\operatorname{median}_{k}\left(F_{k}^{T} \widetilde{Y}_{k}\right)(i, j)
$$

i.e. all the registered frames are fused by using median operator. Then, the problem is reduced to a restoration problem as follows:

$$
\widehat{\mathrm{Z}}=\mathbf{H} \widehat{\mathrm{X}}
$$

where $\hat{X}$ is the estimated version of $\mathrm{X}$ (the HR frame). $\hat{X}$ can be obtained from $\hat{Z}$ by using a restoration step as follows.

$$
\widehat{X}=\underset{x}{\operatorname{argmin}}\left[\|\mathbf{H X}-\hat{\mathrm{Z}}\|_{1}+\lambda\|\mathbf{C X}\|_{1}\right]
$$

The steepest decent solution to the minimization problem in (14) is:

$$
\begin{aligned}
\widehat{\mathrm{X}}^{(\mathrm{n}+1)}=\widehat{\mathrm{X}}^{(\mathrm{n})}- & \beta\left\{\mathbf{H}^{\mathrm{T}} \operatorname{sign}\left(\mathbf{H} \widehat{\mathrm{X}}^{(\mathrm{n})}-\widehat{\mathrm{Z}}\right)\right. \\
& \left.+\lambda \mathbf{C}^{\mathrm{T}} \operatorname{sign}\left(\mathbf{C} \mathrm{X}^{(\mathrm{n})}\right)\right\}
\end{aligned}
$$

where $\beta$ is a scalar representing the step size in the direction of the gradient.

\section{SimULATION RESUlTS}

\section{A. Data Sets}

Two different video sequences including Table Tennis $(352 \times 240$ SIF format) and Football $(352 \times 240$ SIF format $)$ are used to evaluate the proposed algorithm. For these sequences, the YUV components are available. Moreover, we assume that the coloured sequences are already demosaicked or captured by three CCD sensors. In addition to test the proposed modification for the Horn-Schunck optical flow estimation algorithm, we test the optical flow estimation with two famous sequences in the field of optical flow estimation, namely, the rotation and div sequences.

\section{B. Experiment Setup}

In the simulation, we assumed that the available sequences are HR sequences then we generate LR sequences from these sequences by applying blurring, down-sampling operators and adding noise to the HR sequences. Then we used different SR algorithm to reverse these operations.

The LR frames were generated from the original HR video sequences according to the model as in (1), where the frames were blurred by Gaussian operator $(5 \times 5)$ with the variance equal to 1 , down-sampled by a decimation factor equals 2 in the horizontal and vertical directions, and distorted by an additive white Gaussian noise with $30 \mathrm{~dB}$ signal-to-noise ratio.

To demonstrate the efficiency of the proposed modification of the Horn-Shunck optical flow estimation algorithm, simulation results are presented for two different image sequences, with different motion types including rotation and div motions, in comparison with the traditional Horn-Schunck [23], and phase-based [25] optical flow estimation algorithms.

\section{Optical flow Analysis}

Optical flow estimation is one of the main contributions of this work. The efficiency of the proposed modification, which incorporates segmentation with the gradient-based optical flow estimation, is demonstrated as follows.

Fig. 3 shows the results of image sequence that includes rotation motion. Even if phase-based algorithm [25] estimates approximate motion for moving parts, it fails to estimate correct motion at the boundaries as shown in Fig. 3a, where parts of the background are estimated to have high motion.

Fig. $3 b$ shows the results of conventional Horn-Schunck algorithm [23], from this figure, it fails to estimate correct motion at boundaries as shown by estimating motion in the background parts. The main problem of the Horn-Schunck algorithm is treated and solved with the proposed modification s shown in Fig. 3c.

Another example that demonstrates the efficiency of the proposed modification is shown in Fig. 4. This sequence contains div motion. The resulting motion vectors by using phase-based algorithm is scattered and inconsistent for the same region which shows the failure of this algorithm in this sequence as shown in Fig. 4a. Horn-Schunck suffers from the error motion vectors near the boundaries and smooth areas as shown in Fig. 4b. However, the proposed modification has solved this problem as shown in Fig. 4c.

\section{Resolution Enhancement Results}

Fig. 5 shows the results of SR reconstruction for "Table Tennis" sequence. The segmented regions and the bicubicinterpolated frame are shown in Figs. 5a and 5b, respectively. In this figure, the estimated HR frames by incorporating different motion estimation algorithms are shown. From this figure, we can see that as a global motion model, affine motion is not suitable for sequences that contain locally moving objects. This is clear in the disappearance of the ball, as shown in Fig. 5c.

In addition, although conventional optical flow can estimate the flow of each pixel, is very sensitive to noise which results in a noisy edge as shown in Fig. 5d, where the ball is deformed due to the noise effect on the flow estimation process.

On the other hand, segmentation-based optical flow overcomes the problems of the conventional optical flow by assuming a constant motion vector in an arbitrary shaped region (as shown in Fig. 5e).

As another example, the results of Football sequence are shown in Fig. 6. In this figure, the failure of the affine motion is clearer since this sequence contains multi-objects and faster motion as shown in Fig. 6c. In case of using optical flow, the effect of noise is clear at edges, which are not sharp as shown in Fig. 6d. On the other hand, incorporating segmentationbased optical flow estimation preserves sharp edges as shown in Figs. 6e. 


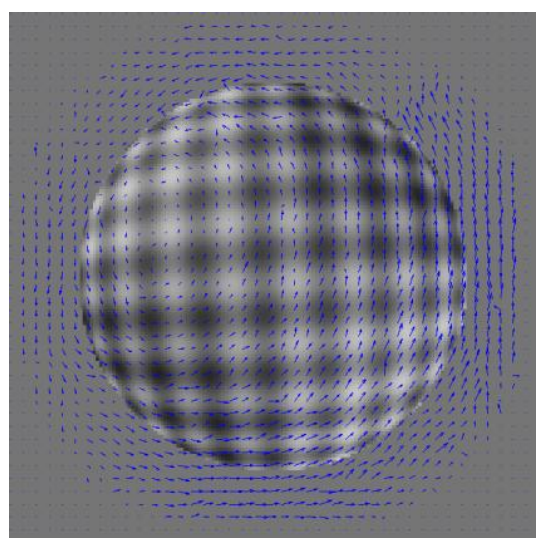

(a)

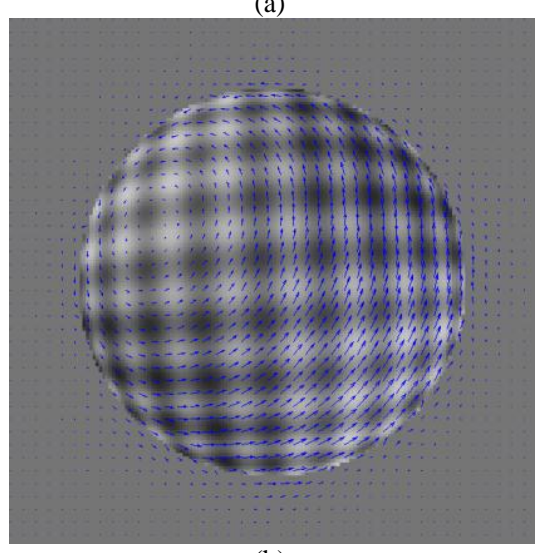

(b)

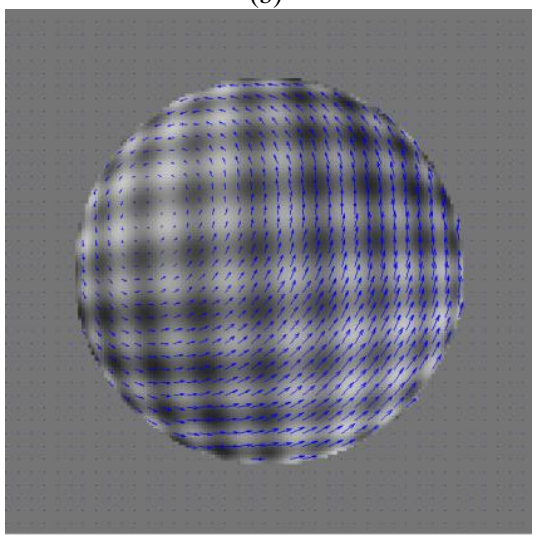

(c)

Figure 3: Image sequence including rotation motion, image with optical flow using; (a) phase-based algorithm [25], (b) conventional Horn-Schunck algorithm [23], and (c) the proposed segmentation -based Horn-Schunck algorithm.

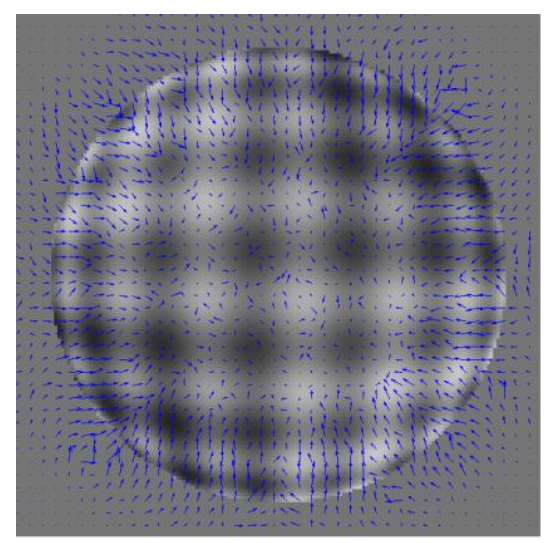

(a)

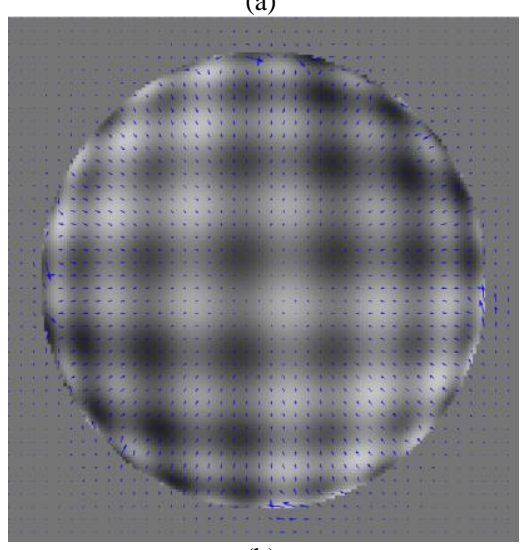

(b)

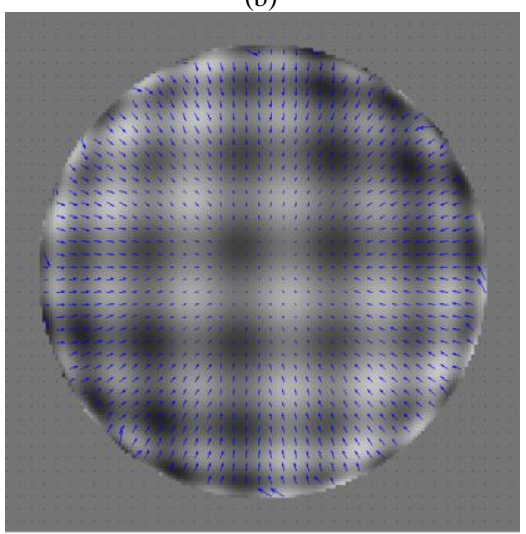

(c)

Figure 4: Image sequence including div motion, image with optical flow using; (a) phase-based algorithm [25], (b) conventional Horn-Schunck algorithm [23], and (c)

segmentation-based Horn-Schunck algorithm. 


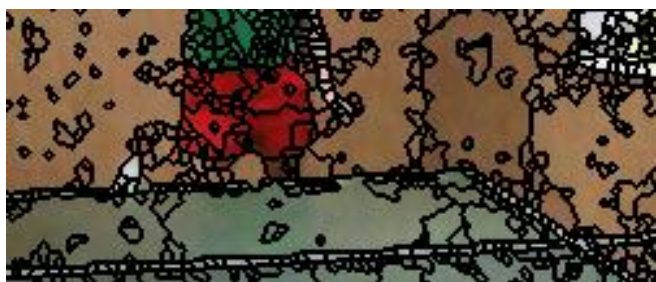

(a)

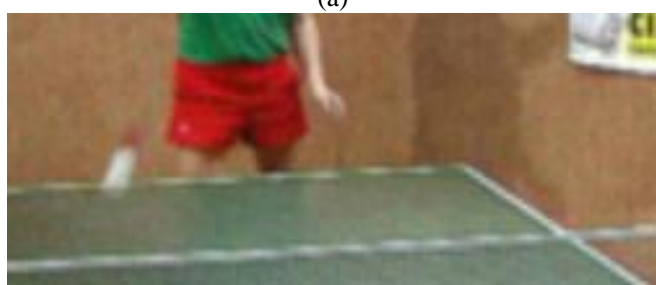

(b)

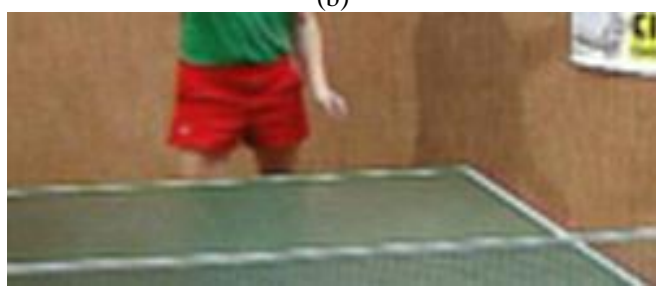

(c)

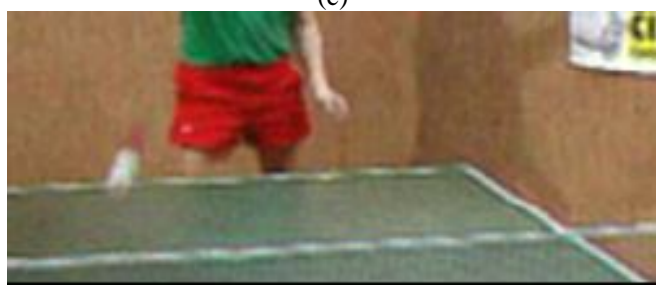

(d)

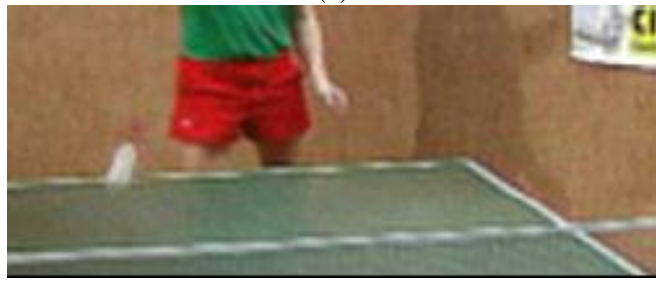

(e)

Figure 5, Table tennis sequence, (a) segmented regions, (b) bicubic interpolation, (c) estimated HR frame by incorporating affine motion estimation, (d) estimated HR frame incorporating conventional optical flow estimation, and (e) estimated HR frame by incorporating modified optical flow estimation.

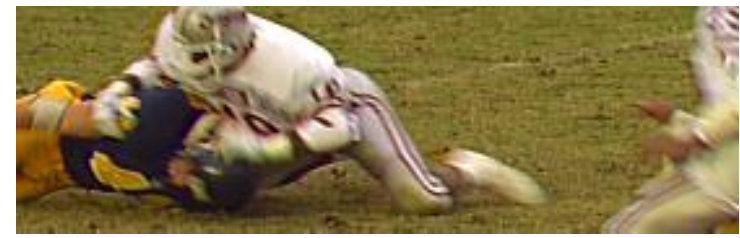

(a)

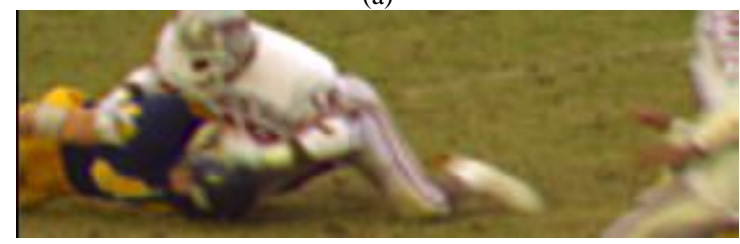

(b)

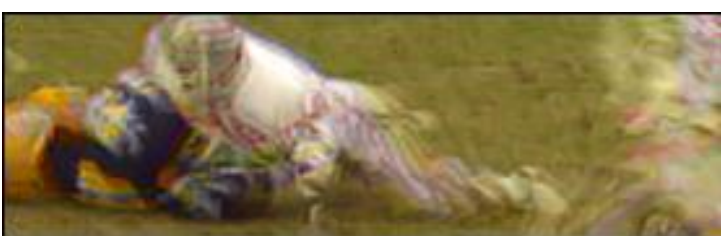

(c)

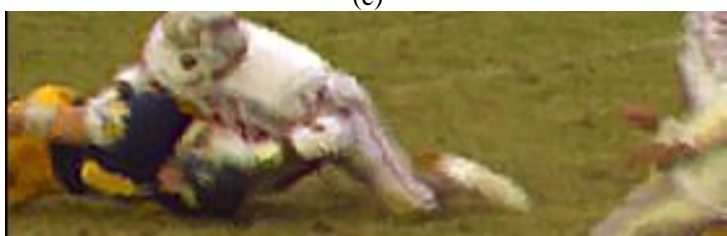

(d)

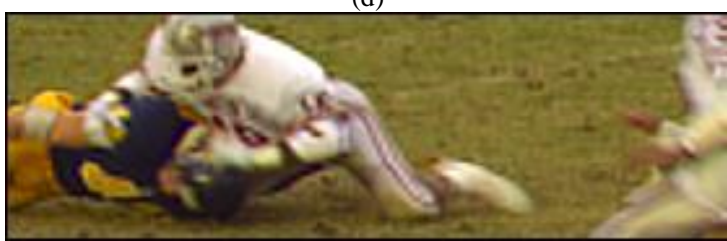

(e)

Figure 6, Football sequence, (a) segmented regions, (b) bicubic interpolation, (c) estimated HR frame by incorporating affine motion estimation, (d) estimated HR frame incorporating conventional optical flow estimation, and (e) estimated HR frame by incorporating modified optical flow estimation.

\section{CONCLUSION}

This paper consists of two main contributions. First, we proposed a modification for the Horn-Schunck optical flow estimation algorithm to overcome problems of conventional gradient-based optical flow estimation algorithms, which are the handling of un-textured regions and the estimation of correct flow vectors near motion discontinuities. We assumed that motion of all pixels in each homogeneous region is the same. Second, we incorporated the proposed segmentationbased optical flow estimation in the resolution enhancement technique. The proposed algorithm overcomes some of the super-resolution problem for video sequences such as the nonrigidity problem where the assumption of general local motion estimates a different motion vector for different parts. The proposed algorithm gave promising results for low resolution sequences with slow/fast motion.

\section{REFERENCES}

[1] Z. Jiang, T.T. Wong and H. Bao, "Practical super-resolution from dynamic video sequences," in Proceedings of IEEE Computer Vision and Pattern Recognition (CVPR 2003), Madison, Wisconsin, USA, June 2003.

[2] S. Farsiu, D. Robinson, M. Elad, and P. Milanfar, "Dynamic demosaicing and color super-resolution of video sequences," Proceedings of the SPIE conference on image reconstruction from incomplete data III, Vol. 5562, October 2004.

[3] M. Elad and Y. Hel-Or, "A fast super-resolution reconstruction algorithm for pure transnational motion and common space invariant blur," IEEE Trans. on Image Processing, Vol.10, no.8, pp.1187-93, August 2001.

[4] S. Farsiu, M. Elad, P. Milanfar, "Multiframe demosaicing and superResolution of color images," IEEE Trans. On Image Processing, Vol. 15, No. 1, January 2006.

[5] S. Farsiu, D. Robinson, M. Elad, and P. Milanfar, "Fast and robust multi-frame super-resolution," IEEE Transactions on Image Processing, vol. 13, no. 10, pp. 1327-1344, October 2004. 
[6] S. Cheol Park, M. Kyu Park, "Super-resolution image reconstruction: a technical overview," IEEE Signal Processing Magazine, May 2003.

[7] W. Zhao, H. Sawhney, "Is super-resolution with optical flow feasible?" Proc. ECCV, vol.1, pp.599-613, 2002.

[8] J. J. Clark, M. R. Palmer, and P. D. Lawrence, "A transformation method for the reconstruction of functions from non-uniformly spaced samples," IEEE Trans. Acoustics Speech, Signal Processing, vol.33, no.4, pp. 1151-1165, 1985.

[9] S. P. Kim, N. K. Bose, and H. M. Valenzuela, "Recursive reconstruction of high resolution image from noisy under-sampled multi-frames,' IEEE Trans. Acoustics Speech, Signal Processing, vol. 38, pp. 1013-1027, June 1990.

[10] M. C. Hong, M. G. Kang, and A. Katsaggelos, "An iterative weighted regularized algorithm for improving the resolution of video sequences," Proc. Int. Conf. Image Processing, vol. 2, pp. 474-477, 1997.

[11] H. Stark and P. Oskoui, "High resolution image reconstruction from lower-resolution image sequences and space varying image restoration," J. Opt. Soc. Am. A, vol. 6, pp. 1715-1726, 1989.

[12] S. Baker and T. Kanade, "Super resolution optical flow," Tech. Rep. CMU-RI-TR-99-36, Robotics Institute, Carnegie Mellon University, Pittsburgh, PA, October 1999.

[13] D. Barreto, L.D. Alvarez, and J. Abad, "Motion estimation techniques in super-resolution image reconstruction a performance evaluation," in Virtual Observatory: Plate Content Digitalization, Archive Mining and Image Sequence Processing, edited by Heron Press, vol. I, 254-268, April 2005.

[14] O. A. Omer and T. Tanaka, "Region-based weighted-norm with adaptive regularization for resolution enhancement," Digital Signal Processing, doi:10.1016/j.dsp.2011.02.005.

[15] O. A. Omer and T. Tanaka, "Extraction of high-Resolution frame from low-resolution video sequence using region-based motion estimation," IEICE Trans. Fundamentals, Vol. E93-A, No.4, April 2010.

[16] H. He, L. P. Kondi, "An image super-resolution algorithm for different error levels per frame," IEEE Trans. Image Process., vol. 15, no. 3, pp. 592-603, March 2006.

[17] R. R. Schultz, R. L. Stevenson, "Extraction of high-resolution frames from video sequences," IEEE Trans. Image Process., vol. 5, no. 6, pp. 996-1011, June 1996.

[18] Hardie, K.J. Barnard, J.G. Bognar, E. Armstrong, and E.A. Watson, "High-resolution image reconstruction from a sequence of rotated and translated frames and its application to an infrared imaging system," Opt. Eng., vol. 37, no. 1, pp. 247-260, 1998.

[19] A. J. Patti, M. I. Sezan, and A. M. Tekalp, "Robust methods for highquality stills from interlaced video in the presence of dominant motion," IEEE Trans. Circuits and Systems for Video Technology, vol. 7, no. 2, pp. 328-342, April 1997.

[20] B. Choi, S.D. Kim and J.B. Ra, "Region-based super-resolution using adaptive diffusion regularization," Opt. Eng., vol. 47, no. 2, pp. 0270061-027006-11, February 2008.

[21] A. van Eekeren, K. Schutte, J. Dijk, D.J.J. de Lange and L.J. van Vliet, "Super-resolution on moving objects and background," in Proc. Int. Conf. Image Process., October 2006, pp. 2709-2712.

[22] P. De Smet and D. De Vleschauwer, "Performance and scalability of highly optimized rain-falling watershed algorithm," Proc. Int. Conf. on Imaging Science, Systems and Technology, CISST98, pp. 266-273, Las Vegas, NV, USA, July 1998.

[23] B. Horn and B. Schunck, "Determining optical flow," Artificial Intelligence, 17:185-203, 1981.

[24] B. Lucas and T. Kanade, "An iterative image registration technique with an application to stereo vision," In International Joint Conference on Artificial Intelligence, pages 674-679, 1981.

[25] T. Gautama, and M.M. Van Hulle, "A phase-based approach to the estimation of the optical flow field using spatial filtering," IEEE Trans. Neural Networks, Vol. 13, No. 5, pp. 1127-1136, 2002.

[26] T. Senst, V. Eiselein, R. H. Evangelio and T. Sikora, "Robust modified L2 local optical flow estimation and feature tracking," Workshop on Applications of Computer Visions, PP. 685 - 690, 2011.

[27] Y. Yang, N. P. Galatsanos, and H. Stark, .Projection based blind deconvolution,. Optical Society of America, vol. 11, no. 9, pp. 2401- 2409, September 1994.

\section{AUthors PROFILE}

Osama A. Omer received his B.Eng. and M.Eng. degrees in electrical engineering from South Valley University, Aswan, Egypt, in 2000 and 2004 respectively. He received his $\mathrm{Ph} . \mathrm{D}$. degree from Tokyo University of Agriculture and Technology in 2009. He is now assistant professor at Aswan University. He spent the summer internship 2008 in Nokia/Tokyo research center. His interests include image/video super resolution and image/video compression. 